\title{
Effectiveness of Structured Physical Activity (PA) Interventions through the evaluation of PA levels, Adoption, Retention, Maintenance and Adherence Rates - A Systematic Review \\ Pre-print article published on: 16 ${ }^{\text {th }}$ August 2019 doi: $10.31236 /$ osf.io/a5fc2
}

Research conducted by the ukactive Research Institute

Article under review in Journal of Physical Activity and Health

Citation: Willinger, N, Steele, J, Atkinson, L, Liguori, G, Jimenez, A, Mann, S. \& Horton, E. (2019). Effectiveness of Structured Physical Activity (PA) Interventions through the evaluation of PA levels, Adoption, Retention, Maintenance and Adherence Rates - A Systematic Review. 10.31236/osf.io/a5fc2 


\section{SUMMARY OF KEY FINDINGS}

- Physical activity (PA) levels in the UK are low with a considerable proportion of the population not meeting recommended levels despite there existing a multitude of interventions aimed at increasing PA.

- Many of these can be classified as structured interventions being that they provide clear recommendations on the prescribed exercise (i.e. frequency of attendance, planned exercise sessions etc.) and are often aimed at increasing the adoption and maintenance of PA behaviours.

- Terms such as adoption, retention, maintenance, and adherence are frequently used terms though their definition varied widely.

- Generally, adoption refers to an individual's uptake of PA at recommended levels, whereas maintenance refers to long-term behaviour change. Retention on the other hand refers to the number of participants in a study available for follow-up assessments, while adherence rates describe the degree to which participants take part in intervention sessions.

- Both retention and adherence are indicators for intervention feasibility. Many structured interventions to increase PA can potentially be delivered through leisure facilities and as retention of participants may also be an important outcome from the perspective of the business model.

- Interventions are rarely assessed for their effectiveness through the measurement of adoption, adherence, retention, or maintenance rates. Thus the aim of this review was to examine the level of reporting of these measures in addition to the evidence for intervention feasibility.

- A systematic review resulted in the identification of 12 intervention studies.

- The evidence suggested that PA interventions appeared to be effective in the short term at increasing PA levels compared to controls, yet results were varying over the longer term.

- Only three studies reported either adoption or maintenance rates which were $67 \pm 16 \%$ and $29 \pm 13 \%$ respectively. Adherence to interventions was $61 \pm 21 \%$ with a retention rate of $75 \pm 13 \%$. 
- Overall, though PA interventions appear to be effective in the short-term at increasing PA levels, there is less evidence to support long term effects and both adoption and maintenance rates are rarely reported. 


\title{
Effectiveness of Structured Physical Activity (PA) Interventions through the evaluation of PA levels, Adoption, Retention, Maintenance and Adherence Rates - A Systematic Review
}

\author{
Willinger $\mathrm{N}^{\mathrm{a}}$, Steele $\mathrm{J}^{\mathrm{b}, \mathrm{c}}$, Atkinson $\mathrm{L}^{\mathrm{d}}$, Liguori $\mathrm{G}^{\mathrm{e}}$, Jimenez $\mathrm{A}^{\mathrm{a}, \mathrm{f}}$, Mann $\mathrm{S}^{\mathrm{g}}$, \& Horton $\mathrm{E}^{\mathrm{a}}$ \\ a Coventry University, Coventry, UK; bukactive Research Institute, London, UK; 'Solent University, Southampton, UK; ${ }^{\mathrm{d} A s t o n}$ University, Birmingham, UK; ${ }^{\mathrm{e}}$ University of Rhode Island, \\ Kingston, RI, USA; ${ }^{\mathrm{f}} \mathrm{GO}$ fit LAB, Ingesport, Madrid, Spain; Places for People Leisure, UK: Contact for Correspondence, Nadja Willinger willingn@uni.coventry.ac.uk
}

\section{SCIENTIFIC ABSTRACT}

Background: Structured physical activity (PA) interventions can potentially be implemented in a variety of facilities, and therefore can reach a large proportion of the population. The effectiveness of interventions is historically evaluated through examination of group differences in outcome measures. Often the proportions of individuals meeting thresholds for PA outcomes related to intervention implementation are not considered. Our aim was to summarise the effectiveness of structured interventions through reported group differences in outcomes, adoption and maintenance rates, and adherence and retention rates, providing information on intervention feasibility. Methods: Database screening resulted in the inclusion of 12 interventions. Results: There was a tendency for structured programmes to result in a significantly greater increase in PA levels than the control conditions in the short-term, with more varying results in the long-term. Only 3 studies published adoption and maintenance rates. On average $67 \pm 16 \%$ (Mean $\pm \mathrm{SD}$ ) of participants were reported as adopting PA, with only $29 \pm 13 \%$ (Mean \pm SD) maintaining this effect. A mean retention rate of $75 \pm 13 \%$ (Mean \pm SD) was observed, and $61 \pm 21 \%$ (Mean $\pm \mathrm{SD}$ ) of intervention sessions were attended as described through adherence rates. Conclusion: Structured interventions were classified as overall effective in short-term on the basis of group differences in PA levels; however, adoption and maintenance rates were rarely reported.

Key words: Efficacy, Feasibility, Dropout, Structured Physical Activity Intervention, Behaviour Change Techniques (BCTs).

\section{INTRODUCTION}

Physical activity (PA) levels in the UK are low, with almost $50 \%$ of the population currently being classified as inactive or insufficiently active, despite a multitude of interventions aiming to increase PA levels ${ }^{1}$. Many of these can be classified as structured interventions as they provide a clear recommendation on the frequency of attendance of pre-planned exercise sessions, amongst other specified components, thus allowing for standardisation and replication ${ }^{2}$. Structured programmes, if proven effective in increasing PA, can potentially be implemented in a large variety of facilities in the private and public health sector, therefore contributing to the global target of reducing inactivity by $10 \%$ by 2025 as defined through the $\mathrm{WHO}^{3}$. Thus, it could be said that this population target is based around concepts of adoption and maintenance of PA.
Adoption and maintenance are frequently used terms, but their definition and measurement in academic literature vary widely ${ }^{4-6}$. Generally, adoption refers to an individual's uptake of PA at recommended levels, whereas maintenance is described as long-term behaviour change $e^{5,6}$. Retention on the other hand refers to the number of participants in a study available for follow-up assessments, while adherence rates describe the degree to which participants take part in intervention sessions $^{7,8}$. Both retention and adherence are indicators for intervention feasibility. Many structured interventions to increase PA can potentially be delivered through leisure facilities and as such a further outcome of importance relating to these behaviours may be the retention of those participants at the leisure centres.

An intervention's effectiveness is commonly assessed via the analysis of mean differences in outcome measures (i.e. PA levels) between treatment groups typically 
through the use of null hypothesis significance testing. However, the extent of its effectiveness is often only expressed as whether a difference between groups meets a threshold for statistical significance. Meaningfulness of this difference is often not considered and arguably this difference better reflects the efficacy of an intervention anyway ${ }^{9}$ Effectiveness instead must consider both the changes in outcome measures in addition to the implementation of interventions within the real world. We propose by reporting the proportion of participants able to achieve and maintain the respectively recommended activity levels, one cannot only get insights on the effectiveness of the intervention but also, in combination with mean changes or differences in outcomes, assess its clinical relevance. Being physically active for 75 to 150 minutes at vigorous or moderate intensity respectively is associated with a multitude of health benefits and analysing the proportion of participants meeting this threshold can be used to infer likelihood that on national and global levels behaviour change might occur and ultimately result in a reduction of healthcare costs and burden of disease associated with inactivity ${ }^{10-13}$.

Reporting adoption and maintenance rates of PA enables readers to compare treatment effects and implementation characteristics between treatment groups and offers more depth than by merely comparing means and measures of variability (i.e. standard deviations). Though of course these sample group level effects may be representative of population effects where studies are adequately powered, and indeed responder counts may merely reflect group mean differences ${ }^{14}$, group level effects only provide limited evidence on the overall effectiveness of an intervention's implementation, particularly for a behavioural outcome such as PA whereby interventions are designed with the intention of increasing PA to meet a particular threshold. Often the proportions of individuals meeting thresholds for outcomes such as PA levels over time are not considered (i.e. adoption, maintenance, retention, or adherence). As such the group level effects seen may not be accurate reflections of the interventions efficacy when implemented in real world settings. This review seeks to address the issue of underreporting of the effectiveness of interventions by summarising the adoption, adherence, retention and maintenance rates of structured PA programmes targeting nonclinical adults. Therefore, the primary aim is to assess, analyse and draw conclusions about the effectiveness of structured PA interventions and programmes in promoting the adoption and maintenance of PA in an insufficiently active adult population. We further aim to draw conclusions on intervention feasibility by summarising adherence and retention rates of participants. The following research questions will be addressed: 1) What proportion of articles on structured PA interventions have evaluated and reported intervention effects for adoption and maintenance?; 2) What are the adoption and maintenance rates achieved by structured exercise programmes aiming to promote an increase in PA levels of insufficiently active adults?; 3) Are structured interventions effective in increasing PA levels compared to a control group or another treatment group? ; and 4) What are the retention and adherence rates of participants of structured interventions and which conclusions on intervention feasibility can therefore be made?

\section{METHODS}

The protocol for this review can be found under the PROSPERO registration number CRD42017061009, and therefore will only be outlined briefly in the following. We followed the PRISMA and AMSTAR 2 guidelines for the conduction and description of this review ${ }^{15,16}$.

\section{Literature Search Strategy}

A combined search of 5 EBSCO databases (MEDLINE, SPORTDiscus, PsycINFO, CINAHL, Academic Search 
Complete) was carried out in addition to separate searches through Scopus and the Cochrane library from the earliest available date until April 2019. The search strategy can be viewed in full on PROSPERO. Terms related to 'adoption', 'intervention', 'physical activity', 'maintenance', 'retention' and 'adults' were combined to identify relevant articles. Terms related to 'nutrition', 'workplace', 'mass media' and 'children' were excluded from the search.

Reference lists of eligible articles were screened for relevant articles. In addition, a separate search of grey literature in the form of evaluation reports of PA programmes in the UK was carried out.

\section{Inclusion Criteria}

The studies and reports had to meet the inclusion criteria detailed below. Only articles published in English language were included. In order to widen the evidence, a pragmatic approach was taken, and this review also includes study designs other than randomised controlled trials e.g. prospective cohort studies. Methodological quality of included articles risk of bias was assessed according to Cochrane Guidelines, as detailed in the next subsection. Inclusion criteria were as follows: 1) The mean age of participants lay between 18-64 years; 2) Participants are characterised by an insufficiently active lifestyle at baseline, defined through activity levels of less than 150 minutes of moderate to vigorous intensity PA per week; 3) The population sampled was healthy adults independent of their weight status; 4) The design is described as a randomised control trial, quasi-experimental trial, or pre- and postintervention i.e. prospective cohort study; 5) The intervention group participated in a structured PA programme, characterised through a recommendation of a defined amount of PA per week achieved through the provision of exercise sessions; 6) Participants were observed for at least 6 months; 7) The primary aim of the intervention(s) was to increase PA levels; 8)
The intervention(s) aimed to change PA behaviour only (single behaviour change); and 9) The intervention(s) did not use mass media and were not described as a homebased or lifestyle intervention.

\section{Data Extraction}

Data were extracted via standardised forms and characteristics of included studies (e.g. type and duration of the intervention(s), follow-up assessments, PA goal or recommendation, applied behaviour change techniques) and study subjects (e.g. gender, age, body mass index (BMI)) were recorded.

Adoption and maintenance rates, and intervention effectiveness expressed as changes in PA levels were defined as primary outcomes. Due to the lack of explicit definitions of the terms 'adoption' and 'maintenance', in this review they were defined as the proportion of participants reaching the respective researcher's recommended PA levels yet with consideration of at what time point this was measured. For the purpose of this review we considered maintenance as a long-term effect measured at least 6 months after adoption (i.e. the first follow-up assessment after the beginning of the intervention). In addition to adoption and maintenance rates, we summarised intervention effectiveness in respect to the reported difference in the magnitude of effects in influencing PA levels between the structured intervention(s) and the control condition.

Within each of the studies the Behaviour Change techniques (BCTs) applied in each intervention were extracted and coded. Two members of the research team independently coded the BCTs on the base of the description of interventions published in each article using the taxonomy of Michie et al. (2013) and discrepancies were resolved through discussions ${ }^{17}$.

\section{Risk of bias}

The Cochrane risk of bias tool was used to detect biases in random sequence 
generation, allocation concealment, blinding of outcome assessors, attrition and reporting ${ }^{18}$. We did not assess bias for blinding study personal and assessors for which intervention a participant received, due to the nature of PA interventions.

For each study, every item was graded into 'high risk' or 'low risk' of bias, or 'unclear' where description of methodology was insufficient. Studies were subjectively graded into high, medium or low risk of bias, considering the types of bias and their severity in addition to the perceived overall methodological complexity. If one item was evaluated as high risk in combination with unclear and low risks for the remaining 4 categories the overall study quality was assumed to be medium, whereas more than 2 categories graded as high risk resulted in a low overall quality of the study. Trials for which the risk of bias due to random sequence generation classified as high were also be given an overall high risk for bias, as this is a potentially strong confounding factor ${ }^{18}$. An overall rating of low risk of bias and therefore high methodological quality was given to studies for which at least 3 categories were classified as low risk in combination with an unclear risk of bias for the remaining 2 items. Any study for which the risk assessment of at least 3 items was not possible due to insufficiently reported methods was be given a moderate overall rating. No studies were excluded due to a poor rating of methodological quality.

\section{RESULTS}

A total of 20,659 articles were identified through database searching. After duplicates were removed 17,008 articles were checked for eligibility through title and abstract screening, resulting in 159 articles analysed through full-text screening, of which two described the same study at different time points ${ }^{19,20}$. After the exclusion of 147 articles which did not meet inclusion criteria the reference lists of the remaining 12 included articles were screened. This search yielded the identification of 5 additional articles, of which one was included in this review, resulting in a final inclusion of 13 papers reporting 12 studies $^{19-}$ 31. The screening process is outlined in the flow chart in Figure 1.

The search of grey literature yielded 8 evaluation reports summarising a multitude of PA programmes in the UK. None of those met all of the inclusion criteria, therefore this information was not included in this review.

\section{Study characteristics}

The characteristics of the 12 included studies are summarised in Table 1. Studies were published between 1982 and 2017 and most commonly conducted in the USA $(50.0 \%)$. The design of the studies was predominantly described as a randomised controlled trial (RCT) (41.2\%), and less than half of included articles were of a high methodological quality $(n=5,41.67 \%)$. Supplementary file 1 provides more information on the risk of bias assessment. A total of 2116 participants of an average age of 49 years were recruited in the studies included in this review. For 4 studies $(33.3 \%)$ there was no age reported. However, these articles were still included in this review as in the methods it was specified participants had to be of an age of 65 or below. Participants in 4 studies $(33.3 \%)$ had a predominantly white background, whereas 3 studies (16.6\%) selectively included Latinos or African Americans $(8.3 \%)$. Half of the studies included women only, and in the remaining 6 articles $58.7 \%$ of participants were female. The interventions were of a mean duration of $10 \pm 5$ months and participants were observed for $14 \pm 6$ months. In 4 studies $(33.3 \%)$ the intervention consisted of a 


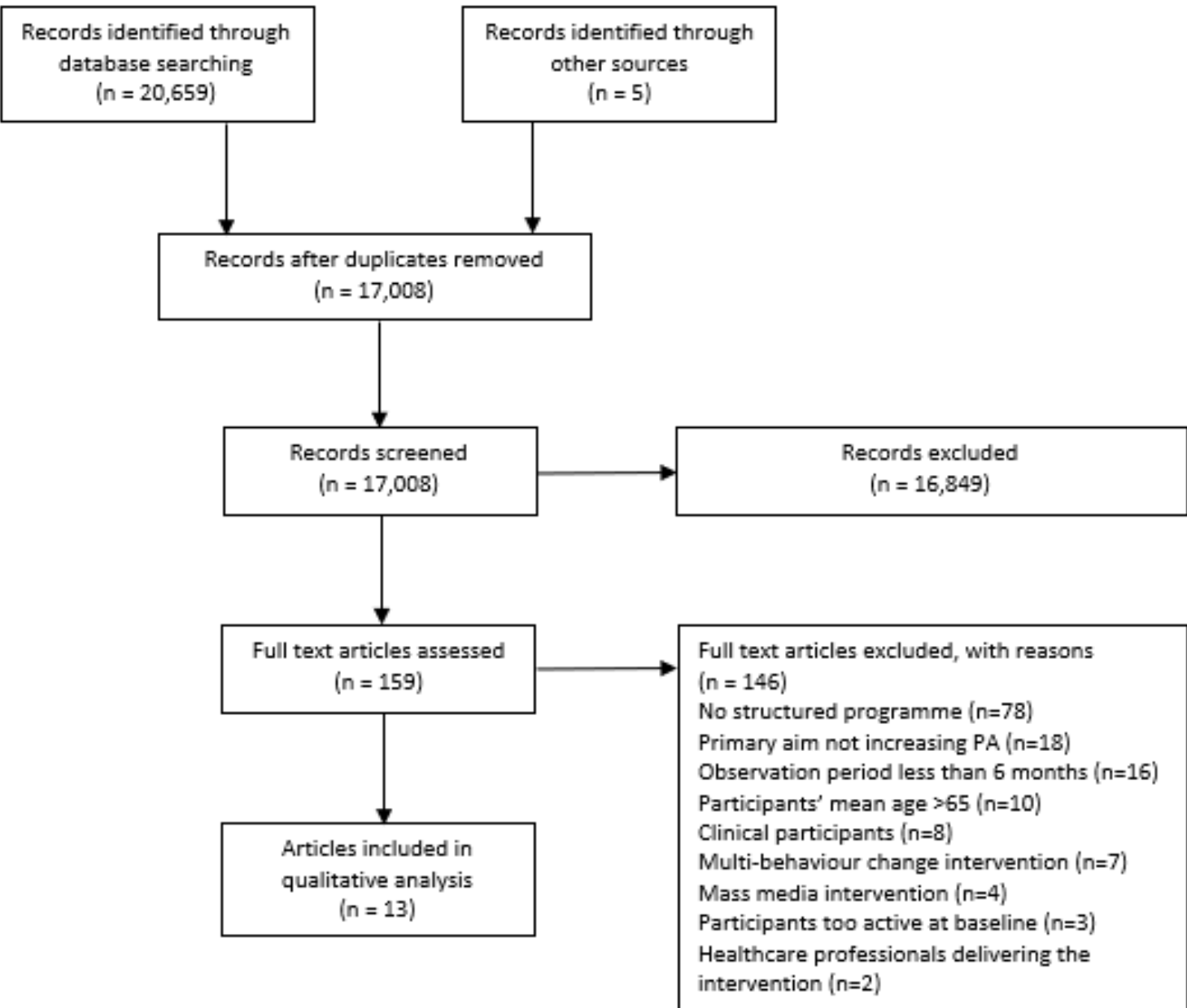

Figure 1. PRISMA Flow Chart for screening process

combination of group sessions and selfdirected PA, 2 interventions were held in a church-setting, a community-setting was chosen by 3 research groups $(25.0 \%)$ and another 2 studies $(16.6 \%)$ started in a group setting but were continued as self-directed PA programmes. Participants were advised to be active ranging from 60 to 270 minutes per week in 1 to 6 supervised and unsupervised sessions. Most interventions were compared to a home-based programme $(n=3,25.0 \%)$ or no treatment control groups $(\mathrm{n}=3,25.0 \%)$.

Interventions applied a mean of $7 \pm 3$ (median=7.5) behaviour change techniques (BCTs), ranging from 3 to 12 , whereas the control condition consisted of 0 to 12 BCTs with a mean of $2 \pm 4$ (median $=0.0$ ). In most studies $(\mathrm{n}=10)$ there were more BCTs applied in the intervention group, but in 2, the same BCTs were applied in the control condition and the structured programme. As all interventions provided exercise sessions, the most frequently used BCTs were 'Instruction on how to perform the behaviour' (4.1; $\mathrm{n}=12)$, 'Demonstration of the behaviour' $(6.1, n=12)$ and 'Behavioural practise/rehearsal' $(8.1 ; n=12)$, followed by 'Goal setting (behaviour)' (1.1, n=8) and 'Graded tasks' (8.7; n=8). The BCTs 'Action planning' (1.4), 'Feedback on outcomes of behaviour' (2.7), 'Social support (practical)' (3.2), 'Social Support (emotional)' (3.3), 'Prompts/Cues' (7.1) and 'Material incentive (behaviour)' (10.1) were applied only once in different studies. Control conditions most frequently used 'Goal setting (behaviour)' (1.1) in addition to the provision of group sessions (BCTs 4.1, 6.1, 8.1). 
Table 1: Study Design and Intervention Characteristics.

\begin{tabular}{|c|c|c|c|c|c|c|c|c|c|c|c|c|c|}
\hline $\begin{array}{c}\text { Author } \\
\text { (Year) }\end{array}$ & Country & Type of Trial & $\begin{array}{l}\text { Study } \\
\text { Quality }\end{array}$ & $\begin{array}{l}\begin{array}{l}\text { Sample } \\
\text { size }\end{array} \\
\end{array}$ & $\begin{array}{l}\text { Age (Years, } \\
\text { Mean } \pm \text { SD) }\end{array}$ & $\%$ Female & Ethnicity & Setting & $\begin{array}{l}\text { Intervention } \\
\text { Duration }\end{array}$ & Recommendation & Control & $\begin{array}{l}\text { BCTs } \\
\text { (Int.) }\end{array}$ & $\begin{array}{l}\text { BCTs } \\
\text { (Cont.) }\end{array}$ \\
\hline $\begin{array}{l}\text { Arredondo } \\
\text { et al. (2017) }\end{array}$ & USA & Cluster-RCT & high & $\begin{array}{l}\text { I: } 187 \\
\text { C: } 183\end{array}$ & $\begin{array}{l}\text { I: } 44.59 .8 \\
\text { C: } 44.4 \pm 9.4\end{array}$ & $100 \%$ & Latino & $\begin{array}{l}\text { Culturally } \\
\text { adapted } \\
\text { church-based } \\
\text { intervention }\end{array}$ & 12 months & $\begin{array}{l}150 \text { minutes MVPA, } \\
\text { individual choice of } \\
\text { the amount of } \\
\text { sessions (Walking } \\
\text { groups, cardio dance } \\
\text { and strength training } \\
\text { classes) }\end{array}$ & $\begin{array}{l}\text { Cancer } \\
\text { screening } \\
\text { classes }\end{array}$ & $\begin{array}{l}1.1 ; \\
1.2 \\
3.1 \\
4.1 \\
6.1 \\
8.1 \\
10.1 \\
10.2 \\
\end{array}$ & NIL \\
\hline $\begin{array}{l}\text { Cox et al. } \\
(2003)\end{array}$ & Australia & RCT & high & $\begin{array}{l}\text { I: } 64 \\
\text { C: } 62\end{array}$ & $\begin{array}{l}\text { I: } 48.0 \\
\text { C: } 48.3 \\
\text { SD not } \\
\text { reported for } \\
\text { I and C }\end{array}$ & $100 \%$ & $\begin{array}{l}\text { Mainly } \\
\text { white }\end{array}$ & $\begin{array}{l}\text { Group-based } \\
\text { programme }\end{array}$ & 18 months & $\begin{array}{l}3 \text { times or more per } \\
\text { week } 20 \text { minutes or } \\
\text { longer MVPA } \\
\text { (walks, aerobics, } \\
\text { circuits) }\end{array}$ & Home-based & $\begin{array}{l}1.1 ; \\
1.2 ; \\
2.1 ; \\
2.2 ; \\
2.3 ; \\
2.4 \\
3.1 ; \\
4.1 ; \\
5.1 ; \\
6.1 ; \\
8.1 ; \\
8.7\end{array}$ & $\begin{array}{l}1.1 ; 1.2 ; \\
2.1 ; 2.2 ; \\
2.3 ; 2.4 \\
3.1 ; \\
4.1 ; \\
5.1 ; \\
6.1 ; \\
8.1 ; 8.7\end{array}$ \\
\hline $\begin{array}{l}\text { De Jong et } \\
\text { al. (2006) }\end{array}$ & $\begin{array}{l}\text { Nether- } \\
\text { lands }\end{array}$ & Cluster-RCT & $\begin{array}{l}\text { moderat } \\
\mathrm{e}\end{array}$ & $\begin{array}{l}\text { I: } 79 \\
\text { C: } 102\end{array}$ & $\begin{array}{l}\text { I: } 59.6 \pm 2.4 \\
\text { C: } 58.8 \pm 2.7\end{array}$ & $\begin{array}{l}\text { I: } 54.4 \% \\
\text { C: } 56.9 \%\end{array}$ & NIL & $\begin{array}{l}\text { Gym-based } \\
\text { leisure-time } \\
\text { programme, } \\
\text { including the } \\
15 \text { most } \\
\text { popular sports } \\
\text { for older adults }\end{array}$ & 6 months & $\begin{array}{l}\text { One session of } 60 \\
\text { min per week }\end{array}$ & Waiting list & $\begin{array}{l}4.1 \\
6.1 \\
8.1\end{array}$ & NIL \\
\hline $\begin{array}{l}\text { Dunn et al. } \\
\text { (1997; } \\
1999)\end{array}$ & USA & RCT & high & $\begin{array}{l}\text { I: } 121 \mathrm{C}: \\
114\end{array}$ & $\begin{array}{l}\text { I: } 46.2 \pm 6.5 \\
\text { C: } 45.9 \pm 6.8\end{array}$ & $\begin{array}{l}\text { I: } 50.9 \% \\
\text { C:50\% }\end{array}$ & $\begin{array}{l}\text { Mainly } \\
\text { white }\end{array}$ & $\begin{array}{l}\text { Group-based } \\
\text { programme, } \\
\text { (after } 6 \text { months } \\
\text { home-based) }\end{array}$ & $\begin{array}{l}6 \text { months ( } 24 \\
\text { months } \\
\text { observation) }\end{array}$ & $\begin{array}{l}150 \text { min MVPA, } \\
\text { stage } 4 \text { or } 5 \text { of the } \\
\text { TTM } \\
\text { initially } 3 \text { sessions, } \\
\text { then } 5 \text { session per } \\
\text { week, duration of } 20- \\
60 \text { min, } 50-85 \% \text { of } \\
\text { maximal aerobic } \\
\text { power }\end{array}$ & Home-based & $\begin{array}{l}1.1 ; \\
1.4 ; \\
2.1 ; \\
3.1 ; \\
4.1 ; \\
6.1 ; \\
8.1 ; \\
8.7\end{array}$ & $\begin{array}{l}1.1 ; 1.2 ; \\
4.1 ; \\
6.1 ; \\
8.1 ; \\
10.3 ;\end{array}$ \\
\hline $\begin{array}{l}\text { Hertogh et } \\
\text { al. (2010) }\end{array}$ & $\begin{array}{l}\text { Nether- } \\
\text { lands }\end{array}$ & RCT & $\begin{array}{l}\text { moderat } \\
\mathrm{e}\end{array}$ & $\begin{array}{l}\text { I: } 78 \\
\text { C: } 68\end{array}$ & $\begin{array}{l}\text { I: } 59.0 \pm 4.5 \\
\text { C: } 58.7 \pm 4.0\end{array}$ & $100 \%$ & NIL & $\begin{array}{l}\text { Group-based } \\
\text { and individual } \\
\text { sessions }\end{array}$ & $\begin{array}{l}12 \text { months } \\
\text { (24 months } \\
\text { observation) }\end{array}$ & $\begin{array}{l}3 \text { sessions per week, } \\
\text { twice supervised ( } 60 \\
\text { min, strength and } \\
\text { endurance), once } \\
\text { individual ( } 30 \text { min } \\
\text { brisk walking or } \\
\text { cycling) }\end{array}$ & $\begin{array}{l}\text { No } \\
\text { intervention }\end{array}$ & $\begin{array}{l}2.3 \\
2.4 \\
4.1 \\
6.1 \\
8.1 \\
8.7\end{array}$ & NIL \\
\hline
\end{tabular}




\begin{tabular}{|c|c|c|c|c|c|c|c|c|c|c|c|c|c|}
\hline $\begin{array}{l}\text { Hovell et al. } \\
(2008)\end{array}$ & USA & RCT & high & $\begin{array}{l}\text { I: } 68 \\
\text { C: } 66\end{array}$ & NIL & $100 \%$ & Latino & $\begin{array}{l}\text { Community } \\
\text { programme for } \\
\text { low-income, } \\
\text { monolingual } \\
\text { Spanish } \\
\text { speaking } \\
\text { Latinas }\end{array}$ & $\begin{array}{l}6 \text { months (12 } \\
\text { months } \\
\text { observation) }\end{array}$ & $\begin{array}{l}3 \text { times } 90 \text { minutes } \\
\text { per week (aerobic } \\
\text { dance exercise) }\end{array}$ & $\begin{array}{l}\text { Safety } \\
\text { education }\end{array}$ & $\begin{array}{l}1.2 ; \\
2.2 ; \\
2.6 ; \\
3.2 ; \\
3.3 ; \\
4.1 ; \\
5.1 ; \\
6.1 ; \\
8.1 ; \\
8.7 ; \\
10.2\end{array}$ & NIL \\
\hline $\begin{array}{l}\text { Kettunen et } \\
\text { al. (2015) }\end{array}$ & Finland & C-ITS & low & $\begin{array}{l}\text { I: } 338 \mathrm{C} \text { : } \\
33\end{array}$ & $\begin{array}{l}\text { I: } 45 \pm 8.8 \\
\text { C: } 41 \pm 6.9\end{array}$ & $\begin{array}{l}\text { I: } 62.72 \% \\
\text { C: } 51.5 \%\end{array}$ & NIL & $\begin{array}{l}\text { Supervised and } \\
\text { individual } \\
\text { exercise } \\
\text { sessions' } \\
\text { (after } 12 \\
\text { months } \\
\text { unsupervised } \\
\text { exercise) }\end{array}$ & $\begin{array}{l}12 \text { months } \\
\text { ( } 24 \text { months } \\
\text { observation) }\end{array}$ & $\begin{array}{l}2 \text { day training camps } \\
\text { at assessments (1-2 } \\
\text { supervised sessions } \\
\text { per } 5 \text { months), } \\
3-5 \text { unsupervised } \\
\text { sessions per week } \\
\text { (walking, skiing, } \\
\text { biking) }\end{array}$ & $\begin{array}{l}\text { No } \\
\text { intervention }\end{array}$ & $\begin{array}{l}2.3 \\
2.4 \\
4.1 \\
6.1 \\
8.1\end{array}$ & NIL \\
\hline $\begin{array}{l}\text { King et al. } \\
(1995)\end{array}$ & USA & RCT & high & $\begin{array}{l}\text { I: } 74 \\
\text { C: } 75\end{array}$ & NIL & $\begin{array}{l}\text { I: } 45.9 \% \text { C: } \\
45.3 \%\end{array}$ & $\begin{array}{l}\text { Mainly } \\
\text { white }\end{array}$ & $\begin{array}{l}\text { Group-based } \\
\text { intervention }\end{array}$ & 12 months & $\begin{array}{l}3 \text { sessions of } 60 \mathrm{~min} \\
\text { per week } \\
\text { (walking/jogging, } \\
\text { treadmills and } \\
\text { stationary cycles) }\end{array}$ & $\begin{array}{l}\text { No } \\
\text { intervention }\end{array}$ & $\begin{array}{l}1.1 \\
4.1 \\
6.1 \\
8.1 \\
8.7\end{array}$ & NIL \\
\hline $\begin{array}{l}\text { Kukkonen } \\
\text { et al. (1982) }\end{array}$ & Finland & Cohort & Low & I: 169 & NIL & $57.4 \%$ & NIL & $\begin{array}{l}\text { Supervised and } \\
\text { individual } \\
\text { sessions, } \\
\text { individual } \\
\text { training } \\
\text { programme for } \\
\text { each } \\
\text { participant }\end{array}$ & 17 months & $\begin{array}{l}\text { 30-60 min 3-6 times a } \\
\text { week (walking, } \\
\text { skiing, jogging, } \\
\text { swimming, cycling), } \\
\text { one a week } \\
\text { supervised } \\
\text { (calisthenics, } \\
\text { volleyball) }\end{array}$ & $\begin{array}{l}\text { No control } \\
\text { group }\end{array}$ & $\begin{array}{l}1.1 ; \\
2.1 ; \\
2.4 \\
4.1 \\
6.1 \\
8.1 \\
8.7\end{array}$ & NIL \\
\hline $\begin{array}{l}\text { Lee et al. } \\
(1997)\end{array}$ & Australia & Cross-over & $\begin{array}{l}\text { moderat } \\
\mathrm{e}\end{array}$ & $\begin{array}{l}\text { I: } 14 \\
\text { C: } 11\end{array}$ & NIL & $100 \%$ & NIL & $\begin{array}{l}\text { Self- } \\
\text { administered } \\
\text { programme of } \\
\text { low-impact } \\
\text { aerobic } \\
\text { exercise, } \\
\text { weekly activity } \\
\text { and education }\end{array}$ & $\begin{array}{l}3 \text { months (12 } \\
\text { months } \\
\text { observation) } \\
*\end{array}$ & $\begin{array}{l}\text { Weekly low intensity } \\
\text { aerobic exercise } \\
\text { session, (duration } \\
\text { unknown, walking or } \\
\text { exercise to music) } \\
2-3 \text { individual } \\
\text { sessions }\end{array}$ & Waiting list & $\begin{array}{l}1.1 ; \\
1.2 ; \\
2.6 ; \\
2.7 \\
4.1 ; \\
6.1 ; \\
8.1 ; \\
8.6 ; \\
8.7\end{array}$ & $2.6 ; 2.7$ \\
\hline $\begin{array}{l}\text { Yang et al. } \\
(2016)\end{array}$ & USA & Pilot RCT & low & $\begin{array}{l}\text { I: } 7 \\
\text { C: } 7\end{array}$ & $\begin{array}{l}\text { I: } 58.4 \pm 6.8 \\
\text { C: } 58.7 \pm 4.1\end{array}$ & $\begin{array}{l}\text { I: } 85.71 \% \\
\text { C: } 85.71 \%\end{array}$ & $\begin{array}{l}\text { Mainly } \\
\text { white }\end{array}$ & $\begin{array}{l}\text { Pilot study, } \\
\text { group-based } \\
\text { yoga } \\
\text { programme } \\
\text { (after } 2 \text { months } \\
\text { home-based) }\end{array}$ & 6 months & $\begin{array}{l}1 \text { supervised session } \\
(90 \text { min) and } 2 \\
\text { unsupervised sessions } \\
\text { at least twice a week }\end{array}$ & $\begin{array}{l}\text { Home-based } \\
\text { (Yoga } \\
\text { DVD), } \\
2 \text { initial } \\
\text { supervised } \\
\text { sessions }\end{array}$ & $\begin{array}{l}1.1 \\
4.1 \\
6.1 \\
8.1 \\
8.6\end{array}$ & $\begin{array}{l}1.1 ; \\
4.1 ; \\
6.1 ; \\
8.1 ; 8.6\end{array}$ \\
\hline
\end{tabular}




\begin{tabular}{|c|c|c|c|c|c|c|c|c|c|c|c|c|c|}
\hline $\begin{array}{l}\text { Young and } \\
\text { Steward } \\
(2006)\end{array}$ & USA & Cluster RCT & high & $\begin{array}{l}\text { I: } 123 \\
\text { C: } 73\end{array}$ & $\begin{array}{l}\text { I: } 48.2 \pm .24 .4 \\
\text { C: } 48.4 \pm 19.7\end{array}$ & $100^{\circ}$ & $\begin{array}{l}\text { African } \\
\text { American }\end{array}$ & $\begin{array}{l}\text { Culturally } \\
\text { adapted } \\
\text { church-based } \\
\text { aerobic } \\
\text { intervention }\end{array}$ & 6 months & $\begin{array}{l}\text { Individualised } \\
\text { activity plans, } \\
60 \text { min per week }\end{array}$ & $\begin{array}{l}\text { alternating } \\
\text { weekly low- } \\
\text { intensity } \\
\text { stretching } \\
\text { classes and } \\
\text { health } \\
\text { lectures }\end{array}$ & $\begin{array}{l}1.1 ; \\
3.2 ; \\
4.1 ; \\
6.1 ; \\
7.1 ; \\
8.1 ; \\
8.7 ; \\
10.2\end{array}$ & 3.2 \\
\hline
\end{tabular}

*Due to the cross-over design of this study only data until 24 weeks were included in this review. BCTs were coded in accordance with the BCT taxonomy published by Michie et al. (2013).

Note: Physical activity (PA); Intervention (I); Control (C); Moderate to vigorous physical activity (MVPA); Metabolic equivalents of tasks per hour (MET/h); transtheoretical model (TTM); Not reported (NIL) 


\section{Change in PA levels}

Effectiveness is commonly assessed through the comparison of interventions to control conditions with respect to whether their effect on increasing PA outcome measures is statistically significantly greater. To assess short-term effectiveness of structured interventions for increasing PA, results at the first follow-up assessment were summarised. Three categories of methods used to define and evaluate PA levels were identified: 1) Changes in objectively measured PA; 2) Changes in an intended outcome of PA behaviour as a proxy (i.e. change in $\mathrm{VO}_{2 \max }$ ); and 3) Changes in self-reported PA levels.

Changes in objectively measured PA levels were assessed by only one study using accelerometery. Three studies examined changes in $\mathrm{VO}_{2 \max }$ through maximal treadmill test as a proxy for PA behaviour (i.e. it was inferred that an increase in cardiorespiratory fitness was indicative of a change in PA levels). Different questionnaires were used to determine selfreported PA levels, e.g. 7-day recalls, the Modified Baecke Questionnaire or the Voorips questionnaire.

On average, the first follow-up assessment took place after 5.92 months (range: 2-12). A total of 7 structured interventions were shown to be more effective than the controls (Table 2). Two trials resulted in an increase in PA outcomes in both treatment groups, indicating the effectiveness of both the control condition and the structured intervention. Only two studies did not result in an effect in either group, of which one was described as a pilot study and was underpowered for statistical analysis.

We did not identify differences in control conditions, PA recommendations, or study quality, between short-term effective and non-effective interventions. All but one of the 6 interventions selectively including women were effective. BCT coding revealed that of 7 effective structured interventions 4 included 'problem solving' (1.2) into their intervention sessions.

To assess long-term changes, we summarised follow-up assessments at least 6 months after short-term effects were assessed. We further distinguished between long term-effects while the intervention was ongoing and after its termination. Of 7 interventions resulting in short-term effects PA levels were assessed in 3 studies while the intervention was ongoing, whereas 3 only evaluated effects after the termination of the intervention. Only one study assessed for effectiveness at both time points. During the intervention period, differences in effects were still significant in 2 out of 4 trials, assessed after an average of 15 months (range: 12-18), whereas after their termination 3 structured programmes sustained higher PA outcomes than the control (21 months; range: 12-24). The number of included studies was too low to be able to identify trends in characteristics of intervention effectiveness in long-term.

\section{Adoption and maintenance rates}

Information on the proportion of participants reaching the PA recommendations from authors within the studies (which notably varied between studies and often diverged from current guidelines; see table 1) was only provided for 3 of 12 articles. One trial published a graph on adoption and maintenance rates, but no information on the exact percentages was provided.

In most cases $(n=10)$, adoption and maintenance behaviour were assessed via the same methods as for physical activity levels, with exemption of 2 studies using activity recalls for the analysis of PA levels, but assessed the stages of change for the evaluation of adoption and maintenance rates. The Stages of Change are also referred to as the 'Transtheoretical Model' and describe a theory aiming to explain behaviour change or somebody's readiness for change. It is based on the assumption each individual moves through 5 stages 


\begin{tabular}{|c|c|c|c|c|c|c|c|c|c|c|c|c|c|}
\hline \multirow[b]{2}{*}{ Author (Year) } & \multicolumn{5}{|c|}{ Adoption } & \multicolumn{5}{|c|}{ Maintenance (during intervention) } & \multicolumn{3}{|c|}{ Maintenance (after intervention) } \\
\hline & Definition & PA Measure & $\begin{array}{l}\text { Time } \\
\text { point }\end{array}$ & Rate & $\begin{array}{l}\text { Short-term } \\
\text { increase in } \\
\text { PA levels }\end{array}$ & Definition & PA Measure & $\begin{array}{l}\text { Time } \\
\text { point }\end{array}$ & Rate & $\begin{array}{l}\text { Long-term } \\
\text { increase in } \\
\text { PA levels }\end{array}$ & $\begin{array}{l}\text { Time } \\
\text { point }\end{array}$ & Rate & $\begin{array}{l}\text { Long-term } \\
\text { increase in } \\
\text { PA levels }\end{array}$ \\
\hline \multicolumn{14}{|c|}{ Objectively measured physical activity } \\
\hline Arredondo et al. (2017) & $\begin{array}{l}\text { Proportion of } \\
\text { participants } \\
\text { reaching 150min } \\
\text { MVPA per week }\end{array}$ & Accelerometer & $\begin{array}{l}12 \\
\text { months }\end{array}$ & $\begin{array}{l}\text { I: } 53 \% \\
\text { C: } \\
40 \%\end{array}$ & $\begin{array}{l}\text { Intervention } \\
\text { group }\end{array}$ & & & $\begin{array}{l}\text { No } \\
\text { follow- } \\
\text { up }\end{array}$ & & & $\begin{array}{l}\text { No } \\
\text { follow- } \\
\text { up }\end{array}$ & & \\
\hline \multicolumn{14}{|c|}{ Physical activity behaviour outcome as proxy } \\
\hline Kukkonen et al. (1982) ${ }^{\text {В }}$ & & $\begin{array}{l}\text { Maximal } \\
\text { Treadmill test } \\
\left(\mathrm{VO}_{2 \max }\right) \\
\text { Maximal } \\
\text { Treadmill test } \\
\left(\mathrm{VO}_{2 \max }\right) \\
\text { Maximal } \\
\text { Treadmill test } \\
\left(\mathrm{VO}_{2 \max }\right) \\
\end{array}$ & $\begin{array}{l}4 \\
\text { months } \\
6 \\
\text { months } \\
2 \\
\text { months }\end{array}$ & NIL & $\begin{array}{l}\text { Intervention } \\
\text { group } \\
\text { Intervention } \\
\text { group } \\
\text { No } \\
\text { difference }\end{array}$ & & $\begin{array}{l}\text { Maximal } \\
\text { Treadmill test } \\
\left(\mathrm{VO}_{2 \max }\right) \\
\text { Maximal } \\
\text { Treadmill test } \\
\left(\mathrm{VO}_{\text {max }}\right) \\
\text { Maximal } \\
\text { Treadmill test } \\
\left(\mathrm{VO}_{2 \max }\right) \\
\end{array}$ & $\begin{array}{l}12 \\
\text { months } \\
17 \\
\text { months }\end{array}$ & $\begin{array}{l}\text { NIL } \\
\text { NIL } \\
\text { NIL }\end{array}$ & $\begin{array}{l}\text { Intervention } \\
\text { group } \\
\text { Intervention } \\
\text { group } \\
\text { No } \\
\text { difference }\end{array}$ & $\begin{array}{l}24 \\
\text { months } \\
\text { No } \\
\text { follow- } \\
\text { up } \\
\text { No } \\
\text { follow- } \\
\text { up } \\
\end{array}$ & NIL & $\begin{array}{l}\text { Intervention } \\
\text { group }\end{array}$ \\
\hline \multicolumn{14}{|l|}{ Stages of Change } \\
\hline Cox et al. $(2003)^{\mathrm{C}}$ & $\begin{array}{l}\text { Stage } 4 \text { of the } \\
\text { TTM (assessed } \\
\text { via Stage of } \\
\text { Change } \\
\text { instrument } \\
\text { Questionnaire }\end{array}$ & $\begin{array}{l}\text { 7-day Recall } \\
\text { (energy } \\
\text { expenditure) }\end{array}$ & $\begin{array}{l}6 \\
\text { months }\end{array}$ & unclear & $\begin{array}{l}\text { Intervention } \\
\text { group }\end{array}$ & $\begin{array}{l}\text { Stage } 5 \text { of } \\
\text { the TTM }\end{array}$ & $\begin{array}{l}\text { Stage of Change } \\
\text { instrument (stage } \\
\text { 5) }\end{array}$ & $\begin{array}{l}18 \\
\text { months }\end{array}$ & unclear & $\begin{array}{l}\text { No } \\
\text { difference }\end{array}$ & $\begin{array}{l}\text { No } \\
\text { follow- } \\
\text { up }\end{array}$ & & \\
\hline $\begin{array}{l}\text { Dunn et al. }(1997 ; 1999) \\
+\end{array}$ & $\begin{array}{l}\text { Meeting ACSM } \\
\text { recommendation } \\
\text { (Stage 4) }\end{array}$ & $\begin{array}{l}7 \text {-day recall } \\
(6 \mathrm{~m}), \\
\text { Maximal } \\
\text { treadmill test } \\
(24 \mathrm{~m})\end{array}$ & $\begin{array}{l}6 \\
\text { months }\end{array}$ & $\begin{array}{l}\text { I: } 85 \% \\
\text { C: } \\
78 \%\end{array}$ & $\begin{array}{l}\text { No } \\
\text { difference }\end{array}$ & $\begin{array}{l}\text { stage } 5 \text { of } \\
\text { the TTM } \\
0\end{array}$ & $\begin{array}{l}\text { Proportion of } \\
\text { participants } \\
\text { meeting the } \\
\text { ACSM } \\
\text { recommendation } \\
\text { (stage 5) }\end{array}$ & $\begin{array}{l}\text { Not } \\
\text { assessed }\end{array}$ & & & $\begin{array}{l}24 \\
\text { months }\end{array}$ & $\begin{array}{l}\text { I: } \\
20 \% \\
\text { C: } \\
20 \%\end{array}$ & No difference \\
\hline \multicolumn{14}{|c|}{ Changes in self-reported physical activity behaviour } \\
\hline De Jong et al. (2006) & & $\begin{array}{l}\text { Voorrips } \\
\text { questionnaire } \\
\text { (energy } \\
\text { expenditure) }\end{array}$ & $\begin{array}{l}6 \\
\text { months }\end{array}$ & NIL & $\begin{array}{l}\text { No } \\
\text { difference }\end{array}$ & & & $\begin{array}{l}\text { No } \\
\text { follow- } \\
\text { up }\end{array}$ & & & $\begin{array}{l}\text { No } \\
\text { follow- } \\
\text { up }\end{array}$ & & \\
\hline \multicolumn{14}{|c|}{ Changes in self-reported physical activity behaviour (continued) } \\
\hline Hertogh et al. (2010) & & $\begin{array}{l}\text { Modified } \\
\text { Baecke } \\
\text { Questionnaire } \\
(\mathrm{MET} / \mathrm{h})\end{array}$ & $\begin{array}{l}12 \\
\text { months }\end{array}$ & NIL & $\begin{array}{l}\text { Intervention } \\
\text { group }\end{array}$ & & $\begin{array}{l}\text { Modified Baecke } \\
\text { Questionnaire } \\
\text { (MET/h) }\end{array}$ & $\begin{array}{l}\begin{array}{l}\text { Not } \\
\text { assessed }\end{array}\end{array}$ & & & $\begin{array}{l}24 \\
\text { months }\end{array}$ & NIL & $\begin{array}{l}\text { Intervention } \\
\text { group }\end{array}$ \\
\hline Hovell et al. (2008) & $\begin{array}{l}\text { Proportion of } \\
\text { participants } \\
\text { reaching 150min } \\
\text { MVPA per week }\end{array}$ & 7-day recall & $\begin{array}{l}6 \\
\text { months }\end{array}$ & $\begin{array}{l}\text { I: } 63 \% \\
\text { C: } \\
16 \%\end{array}$ & $\begin{array}{l}\text { Intervention } \\
\text { group }\end{array}$ & $\begin{array}{l}\text { 150min } \\
\text { MVPA per } \\
\text { week }\end{array}$ & 7-day recall & $\begin{array}{l}\text { Not } \\
\text { assessed }\end{array}$ & & & $\begin{array}{l}12 \\
\text { months }\end{array}$ & $\begin{array}{l}\text { I: } \\
38 \% \\
\text { C: } \\
15 \%\end{array}$ & $\begin{array}{l}\text { Intervention } \\
\text { group }\end{array}$ \\
\hline
\end{tabular}




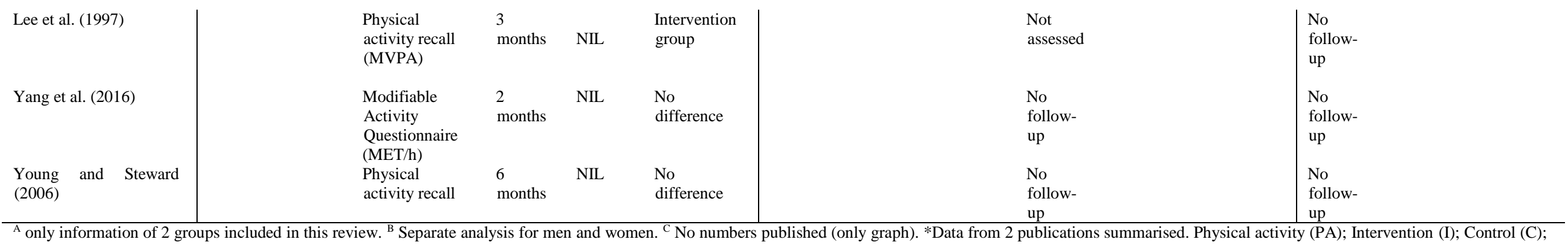

${ }^{\mathrm{A}}$ only information of 2 groups included in this review. ${ }^{\mathrm{B}}$ Separate analysis for men and women. ${ }^{\mathrm{C}}$ No numbers published (only graph). ${ }^{*}$ Data from 2 pu vigorous physical activity (MVPA); Metabolic equivalents of tasks per hour (MET/h); transtheoretical model (TTM); Not reported (NIL) 
when aiming for sustainably change his or her behaviour, namely precontemplation, contemplation, preparation, action, maintenance. Whereas stage 1 and 2 are characterised by someone's intentions to become more active (stage 2) or the absence of such (stage 1), individuals in the preparation stage will undertake first steps towards fulfilling their goal. Stage 4 and 5 describe active individuals who either have (stage 5) or have not (stage 4) been fulfilling their activity goals for at least 6 months ${ }^{32}$. Therefore, a change from stage 1,2 or 3 to stage 4 and from stage 4 to 5 describe adoption or maintenance of PA, respectively.

For structured interventions, a mean adoption rate was reported as $67 \pm 16 \%$ $(n=3)$, whereas on average $45 \pm 31 \%$ participants of the control group were adopting $(n=3)$ (Table 2). This was assessed after 6 and 12 months. Of those, long-term effects were evaluated in 2 cases after 12 or 24 months. The average maintenance rate was at $29 \pm 13 \%(n=2)$ for the intervention group and $18 \pm 3 \%(n=2)$ for the control condition. Due to the low number of studies we were unable to evaluate differences in adoption and maintenance rates and underlying mechanisms.

\section{Retention Rates and Dropout}

Retention rates were published in 9 articles (Table 3). The participation in a structured intervention resulted in a mean retention rate of $75 \pm 13 \%$ as opposed to $68 \pm 11 \%$ in the control group. For 8 studies providing information on reasons for dropout, no differences were found between control conditions and interventions. Six studies reported the frequency of dropout reasons for 136 participants in the control groups and 156 participants in the intervention groups. For the control conditions, most frequently reported reasons for the termination of the participation in the interventions were: Loss to follow-up $(\mathrm{n}=41)$; Lack of time and/or motivation $(n=54)$, and withdrawal, illnesses and/or injuries $(\mathrm{n}=14)$, and non-interest in the study $(n=11)$. Participants in the intervention groups most commonly dropped out due to a lack of time and/or motivation $(n=69)$; loss to follow-up $(n=27)$; withdrawal $(n=20)$; illnesses and/or injuries $(n=14)$; and other unspecified reasons $(n=14)$. For both the intervention and control groups rarely reported reasons included: relocation $(n=3)$; death (of family member) $(\mathrm{n}=2)$; unreliable responses $(n=1)$; pregnancy $(n=1)$. Other reasons where no frequencies were reported were non-participation, lack of spousal support, domestic violence, and missing consent forms.

\section{Adherence Rates}

Adherence was assessed by evaluating the proportion of attended intervention sessions in relation to the recommendation (Table 3). This was documented through self-reported exercise logs and registers at the exercise sessions. In 8 articles adherence to the structured programme was reported, resulting in a mean rate of $61 \pm 21 \%$. In 4 cases, control conditions providing alternative sessions were assessed for adherence, with a mean rate of $59 \pm 30 \%$.

Adherence to the structured programmes was similar between included studies, with the exemption of Young and Steward (2006), who reported an adherence rate of $18 \%^{31}$. For the control conditions Yang et al. (2016) report an adherence rate differing majorly from the average, as participants adhered to $100 \%$ to the activity recommendation, in contrast to the intervention group with $67 \%$.

\section{DISCUSSION}

This review highlights how adoption and maintenance rates of structured PA interventions are rarely reported, yet, alongside reporting of mean changes/differences at group level, are needed to draw more comprehensive conclusions on an intervention's efficacy, effectiveness, and feasibility, and to interpret the relevance of observed effects. 
Table 3: Retention and Adherence Rates.

\begin{tabular}{|c|c|c|c|c|}
\hline Author (Year) & $\begin{array}{c}\begin{array}{c}\text { Length of } \\
\text { Follow-up } \\
\text { (months) }\end{array} \\
\end{array}$ & Retention Rate & Rate & $\begin{array}{l}\text { lherence } \\
\text { Assessment tool }\end{array}$ \\
\hline Arredondo et al. (2017) & 12 & $\begin{array}{l}\text { I: } 84.33 \% \\
\text { C: } 84.93 \%\end{array}$ & Not assessed & \\
\hline Cox et al. (2003) ${ }^{\mathrm{C}}$ & 18 & $\begin{array}{l}\text { I: } 81.25 \% \\
\text { C: } 61.29 \%\end{array}$ & $\begin{array}{l}\text { I: } 65.4 \% \\
\text { C: } 50.8 \%\end{array}$ & $\begin{array}{l}\text { attendance records, } \\
\text { self-report }\end{array}$ \\
\hline De Jong et al. (2006) & 6 & $\begin{array}{l}\text { I: } 48 \% \\
\text { C: } 67 \%\end{array}$ & I: $80 \%$ & $\begin{array}{l}\text { attendance } \\
\text { records }\end{array}$ \\
\hline Dunn et al. $(1997 ; 1999)^{A}$ & 24 & $\begin{array}{l}\text { I: } 78.26 \% \\
\text { C: } 81.97 \%\end{array}$ & Not assessed & \\
\hline Hertogh et al. (2010) & 24 & $\begin{array}{l}\text { I: } 81.25 \% \\
\text { C: } 73.12 \%\end{array}$ & Not assessed & \\
\hline Hovell et al. (2008) & 12 & Not assessed & $\begin{array}{l}\text { I: } 65.8 \% \\
\text { C: } 55.4 \%\end{array}$ & $\begin{array}{l}\text { attendance } \\
\text { records }\end{array}$ \\
\hline Kettunen et al. (2015) & 24 & $\begin{array}{l}\text { I: } 84.85 \% \\
\text { C: } 52.66 \%\end{array}$ & Not assessed & \\
\hline King et al. (1995) ${ }^{\mathrm{A}}$ & 12 & Not assessed & I: $52.6 \%$ & $\begin{array}{l}\text { attendance } \\
\text { records }\end{array}$ \\
\hline Kukkonen et al. (1982) & 17 & $\begin{array}{l}\text { I (men): } 56.94 \% \\
\text { I (women): } 55.67 \%\end{array}$ & Not assessed & \\
\hline Lee et al. (1997) & 12 & $\begin{array}{l}\text { I: } 73.6 \% \\
\text { C: } 72 \%\end{array}$ & I: $80 \%$ & $\begin{array}{l}\text { attendance } \\
\text { records }\end{array}$ \\
\hline Yang et al. (2016) & 6 & $\begin{array}{l}\text { I: } 85.71 \% \\
\text { C: } 57.14 \%\end{array}$ & $\begin{array}{l}\text { I: } 67 \% \\
\text { C: } 100 \%\end{array}$ & exercise logs \\
\hline Young and Steward (2006) & 6 & $\begin{array}{l}\text { I: } 60.98 \% \\
\text { C: } 57.53 \%\end{array}$ & $\begin{array}{l}\text { I: } 18.3 \% \\
\text { C: } 28.6 \%\end{array}$ & $\begin{array}{l}\text { attendance } \\
\text { records }\end{array}$ \\
\hline
\end{tabular}

Though of course these sample group level effects may be representative of population effects, this method only provides limited evidence on the overall effectiveness of an intervention's implementation. As such the group level effects in terms of efficacy seen may not be accurate reflections of the interventions effectiveness when implemented in real world settings due to these behavioural elements. The discrepancy between the assessment of intervention feasibility by considering proportions of individuals adopting and maintaining behaviours, and overall group differences in outcome measures, is highlighted when interpreting the results published by Dunn et al. (1999), who showed that despite a maintenance rate of only $20 \%$, a significant increase in PA levels at follow-up compared to baseline levels was observed ${ }^{19}$. Dunn et al. (1999) applied different questionnaires to assess individual and group effects, which might partly explain the variance of findings, though this further emphasises the need for consistency in reporting. This example does however serve to underline how group level changes may be found to be statistically significant even when only a small proportion of individuals in the sample groups have considerable improvements. Thus, merely considering whether a statistically significant change in a chosen outcome measure such as PA levels occurs does not reflect the relevance and magnitude of effects and ultimately the interventions effectiveness.

The mechanisms of why some PA interventions are effective, whereas others do not result in the anticipated effect are not fully understood. We found a tendency for structured programmes to result in a greater increase in PA levels than the control conditions in short-term, with more varying results in long-term. A meta-analysis found $\mathrm{PA}$ interventions to result in higher PA levels than controls up to 15 months, providing evidence for their effectiveness in long-term ${ }^{33}$. Howlett et al (2019) recently published a meta-analysis including both 
interventions aiming to increase activity levels and reduce sedentary time in healthy adults, and showed that PA interventions were effective in short and long-term ${ }^{34}$. They further conducted meta-regressions, analysing the associations of BCTs influencing intervention effectiveness. A BCT is described as an 'active ingredient' of an intervention, detailing how a targeted behaviour is intended to be changed ${ }^{17}$. Interventions usually consist of a combination of different BCTs and the analysis of patterns in effective or noneffective interventions can therefore contribute to understanding the mechanisms of each intervention ${ }^{35}$. As such, consideration of the BCTs included within interventions may aid in the understanding of which are most effective for enhancing adoption, retention, and maintenance.

Howlett et al. (2019) reported that the BCTs 'Biofeedback' (2.6), 'Demonstration of behaviour' (6.1), 'Behavioural practice/rehearsal' (8.1), and 'Graded tasks' (8.7) were found to be an indicator of intervention effectiveness in short-term, whereas the inclusion of 'problem solving' (1.2), 'Review of behavioural goals' (1.5), and 'Feedback on the behaviour' (2.2) had a detrimental effect $^{34}$. In long-term a larger effect was observed in studies applying the BCTs 'Action planning' (1.4), 'Instruction on how to perform the behaviour'(4.1), 'Prompts/cues' (7.1), 'Behaviour practice/rehearsal' (8.1), 'Graded tasks' (8.7), and 'Self-reward' (10.9), as opposed to the inclusion of 'Information about antecedents' (4.2), which resulted in a smaller effect. Another review identified the BCTs 'goal setting (behaviour)' (1.1) and 'self-monitoring of behaviour' (2.3) as effective for increasing PA levels of overweight and obese adults in both shortand long-term, whereas 'goal setting (outcome) (1.3), 'feedback on outcome of behaviour' (2.7), 'graded tasks' (8.7) and 'adding objects to the environment (12.5) were predictors for long-term effectiveness only ${ }^{36}$.
In our review the application of these BCTs resulted in a large heterogeneity of findings. Due to the low number of included articles however we were unable to formally assess intervention characteristics influencing effectiveness. Of the 8 studies including 'goal setting (behaviour)' (BCT 1.1)', 4 were found to be effective in shortterm, whereas 1 of 4 studies showed longterm effects. The BCT 'Graded tasks' (BCT 8.7) was applied in 6 studies assessing longterm effectiveness, of which 3 showed longterm effectiveness. In 6 studies both BCTs 1.1 and 8.7 were applied, but only one resulted in long-term effectiveness. In regards of self-monitoring of behaviour (2.3), 3 out of 3 studies reported to be effective in short-term and 2 out of 3 in longterm, whereas for self-monitoring of behavioural outcomes (2.4) 3 out of 4 were effective in short term and 2 out of 4 in longterm. The one study applying 'feedback on outcome of behaviour' (BCT 2.7) was found the be effective in short-term, which is accordance to Samdal et al. (2017). Clearly the application of BCTs is at present quite heterogeneous which makes it difficult to draw specific conclusions on which are most effective. To draw clearer conclusions of the effectiveness of specific BCTs applied in structured interventions to increase PA levels, more research empirical research comparing specific approaches to facilitate meta-analysis is warranted.

More information and ultimately more research is needed to systematically summarise adoption and maintenance rates of PA interventions. Of 12 interventions only 3 were assessed for adoption and or maintenance rates. These 3 proved themselves to be successful in inducing adoption in $50-85 \%$ in participants. However, where long-term changes were assessed after the termination of the programmes, only $20-35 \%$ of participants were able to sustain effects and were therefore classified as maintainers. The small number and heterogeneity of studies prevented us from conducting metaanalyses on these rates as intended and we 
were unable to identify intervention components resulting in high adoption and maintenance rates. The low maintenance rates observed in this review indicate the need for future research to improve the effectiveness of interventions in order to increase the proportion of participants meeting the desired behaviours.

The effectiveness of an intervention is further reflected through a participant's retention and adherence rate. In this review, three quarters of participants were available for all follow-up assessments, which in turn can be translated in a dropout rate of approximately $25 \%$ of those who begin the intervention. In a meta-analysis of yoga interventions an average dropout rate of $11 \%$ was observed, rising to $15 \%$ for interventions of a duration of 12 weeks or longer ${ }^{37}$. Similar results have been published by Stubbs et al. (2016) in their meta-analysis, showing that $18 \%$ of participants with depression do not complete the full course of a PA programme $^{38}$. This lies in the recommended range of up to $20 \%$ dropout, as specified by Cochrane guidelines ${ }^{39}$. Although our results show a slightly higher dropout, our findings are in accordance with the dropout rates found in PA interventions with schizophrenia (27\%) and HIV (29\%) patients ${ }^{40,41}$. However, as we only included interventions recruiting healthy individuals, the comparability to these other reviews is limited.

In a review of adherence of cancer survivors to the attendance of exercise sessions, adherence was between $62-78 \%$, whereas for older people this proportion dropped to $58-77 \% \%^{42,43}$. We showed that participants attended on average over $60 \%$ of the recommended exercise sessions. This might have affected the fidelity of the interventions, and the heterogeneity observed in regards to the effectiveness of structured interventions is perhaps likely to be caused by both different intervention designs in addition to non-compliance of participants. Compared to the other included studies, Young and Steward
(2006), reported an adherence rate marginally lower than the average (Aerobic exercise $=18.3 \%$, 'Stretch N Health' $=28.6 \%$ ) 31. In fact, this trial was the only one selectively including African American women, and one of two trials implementing a culturally-adapted church-based programme. As the recommendation of being active for 60 minutes per week lies at the lower end of the spectrum of recommendations in the included studies, this is unlikely to have resulted in the low adherence rate. Historically, African Americans as a demographic are reported to be less active than white adults ${ }^{44,45}$. Common barriers to PA among African American women are lack of motivation, family obligations and lack of social support, and haircare maintenance and the preference of a more voluminous body shape, which are less commonly found in any other ethnic group ${ }^{46}$. This indicates African American Women need to be supported more in becoming and staying physically active, and might explain why adherence in this study was lower compared to the other included articles.

For the control conditions Yang et al. (2016) report an adherence rate differing majorly from the average, as participants adhered $100 \%$ to the activity recommendation, in contrast to the intervention group with $67 \%^{30}$. This suggests the same amount of PA was more easily integrated into an individual's weekly routine when supported at home through a DVD than when delivered face to face through a group-based programme after 2 months. However, the low sample size of this pilot trial does not allow clear conclusions. In both groups the same BCTs were applied.

This review has several limitations. Firstly, we selectively included structured programmes, as they are most commonly applied with respect to PA interventions and, due to their standardisation are more readily replicated ${ }^{2}$. However, while searching for literature, the identification of an intervention as structured was often

Page 17 Pre-print article published on $16^{\text {th }}$ August 2019 (doi: $10.31236 / 0$ sf.io/a5fc2) - The authors confirm they are happy to share this work. 
difficult due to insufficient reporting of methodological design and thus there may be studies that went unidentified and were thus excluded. This insufficient reporting also impaired the risk of bias assessment and the coding for BCTs, although we perceive there to have been an improvement in the descriptions of more recent publications. For each included treatment group, it is possible more BCTs have been applied than we coded for, due to insufficient descriptions of intervention design. We further included academic literature published since 1990, which is possible to have confounded our results as advances in research methods might reduce the comparability between older and more recent publications.

Our ability to draw clear conclusions from this review is impaired by the heterogeneity of structured PA interventions, highlighting the second limitation of this review. Recommended PA levels, the content and delivery of exercise sessions, assessment of PA levels, control conditions, intervention duration and observation period are amongst the multitude of characteristics of studies differing considerably between trials, resulting in a large number of factors potentially influencing intervention effectiveness. Our review was unable to facilitate quantitative synthesis using metaanalysis or permit meta-regression and subgroup analyses taking intervention characteristics into account, therefore we are unable to explore the mechanisms associated with effective structured interventions.

We further highlight the issue of using surrogate measures of PA like maximal treadmill tests for the assessment of effectiveness of PA interventions. Those measures only provide indirect insights on PA levels where it is assumed that, where a $\mathrm{PA}$ intervention is delivered compared to a control, any improvements in cardiorespiratory fitness will only be due to increased PA levels and thus can be used as a surrogate marker of this behaviour. While Page | 18 this might appear reasonable and indeed improvements in cardiorespiratory fitness are an often and intended outcome of performance as a result of PA behaviour being linked to morbidity and mortality ${ }^{47}$, many factors might influence changes in cardiorespiratory fitness including genetics $^{48}$ as well as both the volume and intensity of effort of any PA behaviours ${ }^{49,50}$. As such it is argued for future work that PA behaviours, and indeed the fidelity of any intervention with respect to the PA behaviours (volume, intensity of effort, frequency etc.) should be assessed in addition to the intended outcome of those behaviours (e.g. cardiorespiratory fitness, strength, mental wellbeing etc.). This would permit greater understanding of both what impact interventions have upon PA behaviours and subsequently the degree to which those behaviours might mediate improved health, fitness, and wellbeing.

We originally intended to compare academic literature with grey literature in the form of evaluation reports of structured public health interventions. However, we were unable to identify non-academic literature outlining PA interventions in sufficient detail to be included in this review. We believe evaluation of public health interventions can contribute to the improvement of current knowledge on effective PA interventions, however, lack of control conditions and poor reporting has historically been an issue within the sector ${ }^{51}$, though does seem to have improved and thus this may be a possibility in future reviews ${ }^{52}$.

We perceive our strict inclusion criteria as a strength rather than a limitation, as this enabled us to draw focused conclusions on the effectiveness of structured interventions by reducing heterogeneity of PA programmes. However, we suggest for future reviews to include a broader spectrum of interventions to be able to carry out a more comprehensive review and meta-analysis, and to conduct subanalyses where appropriate. A further strength of this review is our adherence to 
PRISMA and AMSTAR 2 guidelines to produce a review to the recommended standard. Thirdly, we pre-registered the research protocol for this review on PROSPERO prior to conducting the search, enabling researchers conducting similar reviews to understand, reproduce or improve our approach. Moreover, our results can be used to inform future evidence-based structured interventions. On the basis of this review we have developed and are trialling a structured intervention aiming to increase adoption and maintenance rates of members of leisure centres. As part of this research, we are further investigating which factors influence implementation of PA programmes, potentially providing guidelines for academic trials in leisure centres.

\section{CONCLUSION}

From the limited data available it can be concluded that structured interventions are effective in influencing PA levels in the short-term and the adoption of PA behaviours, whereas for maintenance no clear conclusions can be drawn. Implementation of interventions seems to be feasible in short- and long-term, as expressed through moderate adherence and retention rates. However, to assess the effectiveness of structured interventions more information on maintenance rates is needed.

To our knowledge this is the first review aiming to systematically summarise adoption, retention maintenance, and adherence rates of long-term structured PA interventions in non-clinical adults. As these rates are rarely reported, we propose a new point of view in regards of the evaluation of studies considering these, and highly recommend future research to address this issue of underreporting by publishing information on adoption and maintenance rates relative to the recommended amount of PA. This will contribute to the improvement of our understanding of the feasibility of PA interventions, the mechanism through which they are effective in changing PA behaviour, and therefore the design of future PA interventions aiming to tackle global inactivity rates. A more comprehensive summary and meta-analysis of literature is needed, including a wider range of PA interventions.

\section{FUNDING}

This project was funded as part of a joint $\mathrm{PhD}$ studentship (NW) by ukactive and Coventry University

\section{REFERENCES}

1. Sport England. Active people survey. https://www.sportengland.org/research/abo ut-our-research/active-people-survey/. Updated 2016. Accessed 04/21, 2017.

2. Slade S, Dionne C, Underwood M, et al. Consensus on exercise reporting template (CERT): Modified delphi study. Physical Therapy. 2016;96(10):1514-1524.

3. World Health Organisation (WHO). Noncommunicable diseases and mental health; target 3: Reduce prevalence of physical inactivity. http://www.who.int/nmh/ncd-

tools/target3/en/. Updated 2018. Accessed 09/18, 2018.

4. Fjeldsoe B, Neuhaus M, Winkler E, Eakin E. Systematic review of maintenance of behaviour change following physical activity and dietary interventions. Health Psychol. 2011;30(1):99-109.

5. Kahlert D. Maintenance of physical activity: Do we know what we are talking about? Prev Med Rep. 2015;2:178-180.

6. Marcus B, Dubbert P, Forsyth L, et al. Physical activity behavior change: Issues in adoption and maintenance. Health Psychol. 2000;19(1):32-41.

7. Page SJ, Persch AC. Recruitment, retention, and blinding in clinical trials. $A m$ J Occup Ther. 2013;67(2):154-161.

8. Persch AC, Page SJ. Protocol development, treatment fidelity, adherence to treatment, and quality control. Am J Occup Ther. 2013;67(2):146-153.

9. Beedie C, Mann S, Jimenez A, et al. Death by effectiveness: Exercise as 
medicine caught in the efficacy trap! $\mathrm{Br} J$ Sports Med. 2016;50(6):323-324.

10. Friedenreich CM. Physical activity and breast cancer: Review of the epidemiologic evidence and biologic mechanisms. Recent Results Cancer Res. 2011;188:125-139.

11. Löllgen H, Bockenhoff A, Knapp G. Physical activity and all-cause mortality: An updated meta-analysis with different intensity categories. Int $\mathrm{J}$ of Sports Med. 2009;30(3):214-224.

12. Public Health England. Physical inactivity: Economic costs to NHS clinical commissioning groups. https://assets.publishing.service.gov.uk/gov ernment/uploads/system/uploads/attachmen t_data/file/524234/Physical_inactivity_cost s_to_CCGs.pdf. Updated 2016. Accessed 20/04, 2017.

13. Sattelmair J, Pertman J, Ding EL, Kohl III HW, Haskell W, Lee IM. Dose response between physical activity and risk of coronary heart disease: A meta-analysis. Circulation. 2012;124(7):798-795.

14. Atkinson G, Williamson PJ, Batterham

A. Issues in the determination of "responders" and "non-responders" in physiological research. Exp Physiol. 2019; In press.

15. Liberati A, Altman D, Tetzlaff J, et al. The PRISMA statement for reporting systematic reviews and meta-analyses of studies that evaluate healthcare interventions: Explanation and elaboration. BMJ. 2009;339(b2700).

16. Shea B, Reeves B, Wells G, et al. AMSTAR 2: A critical appraisal tool for systematic reviews that include randomised or non-randomised studies of healthcare interventions, or both. BMJ. 2017;358(j4008).

17. Michie S, Richardson M, Johnston M, et al. The behavior change technique taxonomy (v1) of 93 hierarchically clustered techniques: Building an international consensus for the reporting of behavior change interventions. Ann Behav Med. 2013;46(1):81-95.

18. Higgins J, Green S. Cochrane handbook for systematic reviews of interventions Page $\mid 20$ version 5.1.0 [updated march 2011]. The Cochrane Collaboration; 2011.

19. Dunn A, Marcus B, Kampert J, Garcia M, Kohl H, Blair S. Comparison of lifestyle and structured interventions to increase physical activity and cardiorespiratory fitness. JAMA. 1999;281(4):327-334.

20. Dunn AL, Marcus BH, Kampert JB, Garcia ME, Kohl HW,3rd, Blair SN. Reduction in cardiovascular disease risk factors: 6-month results from project active. Prev Med. 1997;26(6):883-892.

21. Arredondo EM, Elder JP, Haughton J, et al. Fe en accion: Promoting physical activity among churchgoing latinas. Am J Public Health. 2017;107(7):1109-1115.

22. Cox KL, Burke V, Gorely TJ, Beilin LJ, Puddey IB. Controlled comparison of retention and adherence in home- vs centerinitiated exercise interventions in women ages 40-65 years: The S.W.E.A.T. study (sedentary women exercise adherence trial). Prev Med. 2003;36(1):17-29.

23. De Jong J, Lemmink KAPM, Stevens M, et al. Six- month effects of the groningen active living model (GALM) on physical activity, health and fitness outcomes in sedentary and underactive older adults aged 55- 65. Patient Educ Couns. 2006;62(1):132-141.

24. Hertogh E, Vergouwe Y, Schuit A, Peeters P, Monninkhof E. Behavioral changes after a 1-yr exercise program and predictors of maintenance. Med Sci Sports Exerc. 2010;42(5).

25. Hovell MF, Mulvihill MM, Buono MJ, et al. Culturally tailored aerobic exercise intervention for low-income latinas. $\mathrm{Am} \mathrm{J}$ Health Promot. 2008;22(3):155-163.

26. KETTUNEN O, VUORIMAA T, VASANKARI T. A 12-month exercise intervention decreased stress symptoms and increased mental resources among working adults - results perceived after a 12-month follow-up. International Journal of Occupational Medicine \& Environmental Health. 2015;28(1):157-168.

27. King AC, Haskell WL, Young DR, Oka RK, Stefanick ML. Long-term effects of varying intensities and formats of physical 
activity on participation rates, fitness, and lipoproteins in men and women aged 50 to 65 years. Circulation. 1995;91:2596-2604.

28. Kukkonen K, Rauramaa R, Siitonen O, Hänninen $\mathrm{O}$. Physical training of obese middle-aged persons. Annals Clin Res. 1982;14(34):80-85.

29. Lee C, White SW. Controlled trial of a minimal-intervention exercise program for middle- aged working women. Psychol Health. 1997;12(3):361-374.

30. Yang K, James KA. Yoga, as a transitional platform to more active lifestyle: A 6-month pilot study in the USA. Health Promot Int. 2016;31(2):423-429.

31. Young DR, Stewart KJ. A church-based physical activity intervention for african american women. Fam Community Health. 2006;29(2):103-117.

32. Marcus BH, Rakowski W, Rossi JS. Assessing motivational readiness and decision making for exercise. Health Psychol. 1992;11(4):257-261.

33. Murray JM, Brennan SF, French DP, Patterson CC, Kee F, Hunter RF. Effectiveness of physical activity interventions in achieving behaviour change maintenance in young and middle aged adults: A systematic review and metaanalysis. Soc Sci Med. 2017;192:125-133.

34. Howlett N, Trivedi D, Troop NA, Chater AM. Are physical activity interventions for healthy inactive adults effective in promoting behavior change and maintenance, and which behavior change techniques are effective? A systematic review and meta-analysis. Transl Behav Med. 2019;9(1):147-157.

35. Michie S, Abraham C, Eccles M, Francis J, Hardeman W, Johnston M. Strengthening evaluation and implementation by specifying components of behaviour change interventions: A study protocol. Implement Sci. 2011;6(10).

36. Samdal GB, Eide GE, Barth T, Williams G, Meland E. Effective behaviour change techniques for physical activity and healthy eating in overweight and obese adults; systematic review and meta-regression analyses. Int $J$ Behav Nutr Phys Act. 2017; 14(42).

37. Cramer H, Haller H, Dobos G, Lauche R. A systematic review and meta-analysis estimating the expected dropout rates in randomized controlled trials on yoga interventions. Evid Based Complement Alternat Med. 2016;2016:5859729.

38. Stubbs B, Vancampfort D, Rosenbaum $\mathrm{S}$, et al. Dropout from exercise randomized controlled trials among people with depression: A meta-analysis and meta regression. J Affect Disord. 2016;190:457466.

39. Furlan AD, Pennick V, Bombardier C, van Tulder M, Editorial Board, Cochrane Back Review Group. 2009 updated method guidelines for systematic reviews in the cochrane back review group. Spine (Phila Pa 1976). 2009;34(18):1929-1941.

40. Vancampfort D, Mugisha J, Richards J, et al. Dropout from physical activity interventions in people living with HIV: A systematic review and meta-analysis. AIDS Care. 2017;29(5):636-643.

41. Vancampfort D, Rosenbaum S, Schuch FB, Ward PB, Probst M, Stubbs B. Prevalence and predictors of treatment dropout from physical activity interventions in schizophrenia: A meta-analysis. Gen Hosp Psychiatry. 2016;39:15-23.

42. Kampshoff C, van Mechelen W, Schep $\mathrm{G}$, et al. Participation in and adherence to physical exercise after completion of primary cancer treatment. Int J Behav Nutr Phys Act. 2016;13(100).

43. Picorelli A, Pereira L, Pereira D, Felício D, Sherrington C. Adherence to exercise programs for older people is influenced by program characteristics and personal factors: A systematic review. $J$ Physiother. 2014;60(3):151-156.

44. American Heart Association. Heart Disease and Stroke Statistics-

2018 Update: A report from the American Heart Association. Circulation. 2018;137(e67):e492.

45. American Heart Association. Heart Disease and Stroke Statistics- 
2006 Update.

Circulation 2006;113(6):e85-e151.

46. Joseph RP, Ainsworth BE, Keller C, Dodgson JE. Barriers to physical activity among african american women: An integrative review of the literature. Women Health. 2015;55(6):679-699.

47. Ross R, Blair SN, Arena R, et al. Importance of assessing cardiorespiratory fitness in clinical practice: A case for fitness as a clinical vital sign: A scientific statement from the american heart association. Circulation. 2016;134(24):e653-e699.

48. Peter I, Papandonatos GD, Belalcazar $\mathrm{LM}$, et al. Genetic modifiers of cardiorespiratory fitness response to lifestyle intervention. Med Sci Sports Exerc. 2014;46(2):302-311.

49. Montero D, Lundby C. Refuting the myth of non-response to exercise training: 'Non-responders' do respond to higher dose of training. J Physiol. 2017;595(11):33773387.

50. Ross R, de Lannoy L, Stotz PJ. Separate effects of intensity and amount of exercise on interindividual cardiorespiratory fitness response. Mayo Clin Proc. 2015;90(11):1506-1514.

51. Public Health England. Identifying what works for local physical inactivity interventions.

http://researchinstitute.ukactive.com/downl oads/managed/Identifying_what_works.pdf . Updated 2014. Accessed 14/03, 2018.

52. ukactive. Moving at Scale: Promising practice and practical guidance on evaluation of physical activity programmes in the

UK. http://research.ukactive.com/moving-atscale/ Accessed 14/03, 2018 